\title{
Using Benders Decomposition for Solving Ready Mixed Concrete Dispatching Problems
}

\author{
Mojtaba Maghrebi $^{a}$, Vivek Periaraj $^{b}$, S. Travis Waller ${ }^{a, c}$, Claude Sammut $^{d}$ \\ ${ }^{a}$ School of Civil and Environmental Engineering, The University of New South Wales (UNSW), Sydney, Australia \\ ${ }^{\mathrm{b}}$ Department of Systems and Industrial Engineering, The University of Arizona, Tucson, AZ, USA \\ ${ }^{\mathrm{c}}$ National Information and Communications Technology Australia (NICTA), Sydney, Australia \\ ${ }^{\mathrm{d}}$ School of Computer Science Engineering, The University of New South Wales (UNSW), Sydney, Australia \\ E-mail: $\{$ maghrebi, s.waller, c.sammut\}@unsw.edu.au, vivek.periaraj@gmail.com
}

\begin{abstract}
Large scale dispatching problems are technically characterized as classical NP-hard problems which means that they cannot be solved optimally with existing methods in a polynomial time. Benders decomposition is recommended for solving large scale Mixed Integer Programming (MIP). In this paper we use the Bender Decomposition technique for reformulating the Ready Mixed Concrete Dispatching Problem (RMCDP). Benders decomposition involves separating the original RMCDP formulation into the master (lower bound) and sub-problems (upper bound). The master problem only deals with integer variables and the sub problem is usually a linear programming problem. Benders optimally cuts and Benders feasibility cuts are added to the master problem upon solving the sub-problem at each iteration.. The proposed method is tested on a single real instance and results are reported.
\end{abstract}

Keywords: Benders Decomposition; Ready Mixed Concrete (RMC), Dispatching

\section{Introduction}

During the past 10 years a growing body of literature has been devoted to Ready Mixed Concrete Dispatching Problems (RMCDP); however, this area still suffers from a lack of practical solutions [1-6]. In RMCDP it is desirable to find the best truck and depot allocation for each delivery. A few attempts have been made to acquire the exact solution of RMCDP; nevertheless, as a result of increasing the size of the problem the complexity is increased exponentially [4] and cannot be solved in a polynomial time. Heuristic solutions have been implemented widely in the literature to alleviate this problem. Among the introduced methods, Genetic
Algorithms is the most promising heuristic solution in the RMCDP literature [1, 3, 5, 7-10]. Other heuristic methods also have been tested in this context, such as Ant Colony [11], Particle Swarm Optimization (PSO) [12, 13], Bee Colony Optimization (BCO) [14] and Tabu Search (TS) [14]. Despite developments in implementing heuristic methods in RMCDP, the solution structure of most of the mentioned techniques is pretty much same. Moreover, the main drawback for these techniques is that there are a number of infeasible allocations in the outcomes of these techniques. Thus, via supplementary algorithms, obtaining a viable solution has been attempted. To overcome this issue, []ㅡ presented an evolutionary based method which can solve the RMCDP without the need for any supplementary algorithm.

Rather than simply looking at heuristic methods some other numerical approaches have been studied. Yan, Lai [15] introduced a numerical method for solving the RMC optimization problem. They proposed a method that works by cutting the solution space iteratively and as well is integrated with branch-and-bound. Lin, Wang [16] introduced a new RMCDP formulation inspired by the job shop problem. Yan, Lin [17] used decomposition and relaxation techniques coupled with a mathematical solver to solve the problem. Variable Neighbourhood Search (VNS) was tested by Payr and Schmid [18] to deal with RMCDP. One of the robust RMCDP formulations was proposed by Asbach, Dorndorf [19]. In this method, depots and customers are divided into sub-depots and sub-customers. More recently, Maghrebi, Periaraj [20] implemented a Column Generation (CG) method which is amenable to the Dantzig-Wolfe reformulation for solving large scale models which with available computing facilities cannot optimally solve in polynomial time. However, the Benders decomposition [21] has not been used in RMCDP, which is the main contribution of this paper. 


\section{RMC Benders Decomposition}

In 1962 [21] Benders introduced a decomposition method for solving MIP which later was generalized by Geoffrion [22]. Benders' methodology involves decomposing the compact formulation into master (lower bound) and sub-problems (upper bound). The master problem is usually an integer programming problem and the sub-problem is usually a linear programming problem. In each iteration, the sub-problem is solved for a given solution of the master problem. If the sub-problem is optimal, then an extreme dual solution is used to form what is called a Benders optimality cut and added to the master problem. If the sub-problem is primal infeasible (or dual unbounded), then an unbounded extreme dual ray is used to form what is called a Benders feasibility cut and added to the master problem. The master and sub-problems are solved iteratively in this way until the bounds are strengthened and the algorithm converges.

A few RMCDP formulations have been introduced, such as $[\underline{5}, \underline{15-17}, \underline{19}, \underline{23-25]}$. To simplify the formulation in some methods $[\underline{15}, \underline{17}, \underline{19}, \underline{26}]$ the depots and customers are divided into a set of sub-depots and sub-customers, respectively based on the number of loads at depots and the number of required deliveries. The compact formulation of RMCDP can be stated as follows $[\underline{4}, \underline{19}]$ if we assume RMCDP as a graph $G=(V, E)$ in which $V$ is the set of vertices belonging to start points, customers, depots and end points $V=\left\{u_{s} \cup C \cup D \cup v_{f}\right\}$. Additionally, $E$ is the set of edges belonging to the distance between vertices.

$$
\text { Minimize } \sum_{u} \sum_{v} \sum_{k} z_{u v k} x_{u v k}-\sum_{c} \beta_{c} y_{c}
$$

Subject to:

$$
\begin{aligned}
& \sum_{u \in u_{s}} \sum_{v} x_{u v k}=1 \forall k \in K \\
& \sum_{u} \sum_{v \in v_{f}} x_{u v k}=1 \forall k \in K \\
& \sum_{u} \sum_{v} x_{u v k}-\sum_{v} \sum_{j} x_{v j k}=0
\end{aligned}
$$

$\forall k \in K, v \in C \cup D$

$$
\sum_{u \in D} \sum_{k} x_{u v k} \leq 1 \forall v \in C
$$

$$
\begin{aligned}
& \sum_{v \in C} \sum_{k} x_{u v k} \leq 1 \forall u \in D \\
& \sum_{u \in D} \sum_{k} q_{k} x_{u v k} \geq q_{c} y_{c} \forall c, v \in C \\
& -M\left(1-x_{u v k}\right)+s_{u}+t_{u v k} \leq w_{v}-w_{u} \\
& M\left(1-x_{u v k}\right)+\gamma+s_{u} \geq w_{v}-w_{u} \quad \forall(u, v, k) \in E \\
& x_{u v k} \in\{0,1\} \wedge y_{c} \in\{0,1\} \quad \forall(u, v, k) \in E
\end{aligned}
$$

In the RMCDP, the master problem consists of customer only constraints, depot only constraints, demand constraints, delivery constraints and perishability constraints involving only depot to customer arcs. The sub-problem consists of truck start constraints, truck finish constraints, customer flow constraints, depot flow constraints, time truck start constraints, time return constraints and time truck finish constraints.

\subsection{Benders Master Problem:}

The master problem in RMCDP is a mixed integer programming model and involves assignment of depots and customers subject to the time requirements. The Benders master problem can be formally stated as follows:

Minimize $\sum_{u} \sum_{v} \sum_{k} z_{u v k} x_{u v k}-\sum_{c} \beta_{c} y_{c}+Z$

Subject to:

$\sum_{u \in u_{s}} \sum_{k} x_{u v k} \leq 1 \forall v \in C$

$\sum_{v \in C} \sum_{k} x_{u v k} \leq 1 \forall u \in D$

$\sum_{u \in D} \sum_{k} q_{k} x_{u v k} \geq q_{c} y_{c} \forall c, v \in C$ 
$-M\left(1-x_{u v k}\right)+s_{u}+t_{u v k} \leq w_{v}-w_{u}$

$$
\forall(u, v, k) \in E
$$

$Z=\sum_{k \in K} Z_{k}$

$w \in \Phi_{k}, y \in \Omega_{k} \forall k \in K$

$u \in D, v \in C, k \in K$

\subsection{Benders Sub-problem}

The sub-problem in RMCDP is an integer programming model. It can be solved as Minimum Cost Flow network problem that enables to exploit the integrality properties and so can be solved as a linear programming model. The Benders sub-problem can be formally stated as follows:

Minimize $\sum_{u} \sum_{v} \sum_{k} z_{u v k} x_{u v k}$

Subject to:

$\sum_{u \in u_{s}} \sum_{v} x_{u v k}=1 \forall k \in K$

$\sum_{u} \sum_{v \in v_{f}} x_{u v k}=1 \forall k \in K$

$\sum_{u} \sum_{v} x_{u v k}-\sum_{v} \sum_{j} x_{v j k}=0$

$\forall k \in K, v \in C \cup D$

$0 \leq x_{u v k} \leq 1$ if $x_{u v k}$ isfeasible

$0 \leq x_{u v k} \leq$ oif $x_{u v k}$ isnotfeasible

$u \in C, v \in D, k \in K$

$u \in u_{s}, v \in D, k \in K$

$$
u \in C, v \in v_{f}, k \in K
$$

\section{Solution Approach}

The master problem is a mixed integer problem and can be solved using branch-and-cut [27]. The master problem can be considered as an assignment problem of depot to customer arcs, subject to demand constraints and time restrictions. The sub-problem is solved at the truck level for a given set of optimal assignments associated with a given truck from the master problem. The optimal depots to customer arcs from the master problem determine the bounds of the customer service times at given customer locations. The delivery constraints affect the lower bound, while the perishability constraints affect the upper bound of the customer time. The start to depot arcs, customer to depot arcs and customer to finish arcs in the sub-problem are adjusted for their bounds based on the new bounds of the customer time obtained from the optimal solution of the master problem. The prerequisite for the sub-problem in the Benders solution framework is that it needs to be a linear programming model, such that its weak duality property can be used to derive the Benders optimality and feasibility cuts. The sub-problem can be solved either by the network simplex method [28] or by using the LP optimizer (primal or dual) to obtain the extreme dual solution or extreme unbounded dual ray solution.

The following two integrality properties motivate solving the sub-problem using the network simplex optimizer or the LP optimizer (primal or dual). The first type of integrality property can be formally stated as follows:

$$
\operatorname{Co}\{x \in X \mid A x \leq B\}=\operatorname{Co}\{x \in\{0,1\} \vee A x \leq B\}
$$

and occurs when the optimal objective of the linear programming relaxation with real solution is same as the optimal objective with integer solution. The second type of integrality property can be formally stated as follows:

$$
\text { minimize }\{c x: A x=b, l \leq x \leq u\}
$$

and is bounded from below on the feasible region; if the problem has a feasible solution, and the vectors $b, l$ and $u$ are integers, then the problem has at least one integer optimum solution.

\subsection{Optimality Cuts}

From an extreme dual solution of the sub-problem, the following Benders optimality cut is added to the master problem. Let,

$\Pi_{k}$ be the extreme dual associated with the truck start constraint (2) for the truck $k$.

$\lambda_{k}$ be the extreme dual associated with the truck finish constraint (3) for the truck $k$.

$\theta_{d k}$ be the extreme dual associated with the depot flow constraint (4) for the truck $k$.

$\theta_{c k}$ be the extreme dual associated with the customer flow constraint (4) for the truck $k$.

$\sigma_{k}$ be the sum of extreme duals associated with bound constraints of the arcs in the sub-problem for the truck $k$.

$$
\begin{aligned}
\theta_{u k} \times x_{u v k}-\theta_{v k} & \times x_{u v k}+Z_{k} \\
\geq & \left(\pi_{k}-\lambda_{k}\right)+\sigma_{k} \\
& \forall u \in D, v \in C, k \in K
\end{aligned}
$$

Each optimality cut for a given truck $k$ is added to the set $\Omega_{k}$. The convergence of the algorithm is defined by 
the value of the variable $Z$. This variable is sometimes called the approximation variable or the recourse function in the Benders decomposition context and is a measure of the dual objective of the sub-problem. As each optimality cut added to the master problem attempt to improve the value of this bound and thus convergence of algorithm can be stated when the value of $Z$ is equal to or within a specified tolerance of the objective value of the sub-problem.

\subsection{Feasibility Cuts}

From an unbounded dual ray solution of the sub-problem, the following Benders feasibility cut is added to the master problem. Let,

$\pi_{\mathrm{k}}$ be the extreme ray associated with the truck start constraint (2) for the truck $\mathrm{k}$.

$\lambda_{\mathrm{k}}$ be the extreme ray associated with the truck finish constraint (3) for the truck $\mathrm{k}$.

$\theta_{\mathrm{dk}}$ be the extreme ray associated with the depot flow constraint (4) for the truck k.

$\theta_{\mathrm{ck}}$ be the extreme ray associated with the customer flow constraint (34) for the truck $\mathrm{k}$.

$\sigma_{\mathrm{k}}$ be the sum of extreme rays associated with the bound constraints of the arcs in the sub-problem for the truck $\mathrm{k}$.

$\theta_{\mathrm{uk}} \times \mathrm{x}_{\mathrm{uvk}}-\theta_{\mathrm{vk}} \times \mathrm{x}_{\mathrm{uvk}} \geq\left(\pi_{\mathrm{k}}-\lambda_{\mathrm{k}}\right)+\sigma_{\mathrm{k}}$

$$
\forall \mathrm{u} \in \mathrm{D}, \mathrm{v} \in \mathrm{C}, \mathrm{k} \in \mathrm{K}
$$

Each feasibility cut for a given truck $\mathrm{k}$ is added to the set $\phi_{\mathrm{k}}$

This process is terminated when the model converges.

\section{Case Study}

The proposed Benders decomposition is tested by field data which belong to an active RMC with three active depots and around 50 trucks. From the available dataset, the data from a day on which 22 customers were to be supplied was selected for further studies. Among the customers, 2 needed 3 deliveries and 4 needed 2 deliveries, while the remainder only needed 1 delivery. The authors believe that this instance is not a very complex RMCDP problem; however, the main goal in selecting a small instance is to provide an opportunity to investigate all aspects of RMC resource allocations in detail.

The algorithm was developed in $\mathrm{C}++$ and tested on a RedHat(R) CentOS(R)5.9 Linux server with $83.60 \mathrm{GHz}$ Intel(R) Xeon(R) CPUs and a 188 GB physical memory. The IBM CPLEX тм version 12.5.0.0 with parallel optimizers using up to 8 threads was used in the study.

The most important criteria in optimization is the value of the objective function. In Figure 1 the trend of the objective function inclusive of $Z$ is shown and in Figure 2 the best solution obtained so far over iterations is illustrated. As mentioned above, computing time is a challenge for RMCDP. Figure 3 focuses on this issue by depicting the cumulative elapsed time over the iterations.
Now, the obtained solution will be discussed. According to the RMCDP the graph $G$ includes 4 types of arcs:

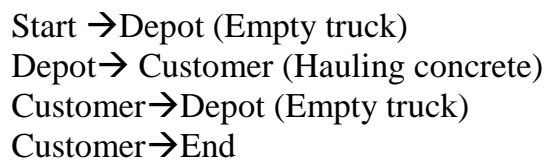

The models must find feasible arcs for all assigned trucks at minimum cost. The obtained solutions are shown in Figure 4 and Figure 5. In these two figures, the blue dots are depots and red dots are customers. An arc that connects a depot to a customer also represents the travel distance between these two locations. The thickness of an arc shows the number of trucks using that route, and similarly the size of red dots (customers) represents the number of required deliveries.

Figure 5 similarly shows the travel distance between customers (after unloading the concrete) to depots for loading fresh concrete.

In the test instance, 17 trucks were available and the model used all of them (Figure 6). Although there is the possibility of using fewer trucks to serve all customers, minimizing the number of trucks has not been associated with the objective function.

\section{Conclusion}

The application of Benders' decomposition to the Ready Mixed Concrete Dispatching Problem (RMCDP) has been studied in this paper. Optimally solving larger scales of RMCDP was the main motivation for this approach. Benders decomposed the original RMCDP formulation into the master (lower bound) and subproblems (upper bound). In the master problem only discrete variables are dealt with, and the sub-problem is usually a linear programming problem. A Benders optimal cut is added to the model in each iteration, if the sub-problem obtained a feasible solution. Also, a Benders feasibility cut is added if the sub-problem is unbounded or infeasible. This process is iteratively continued until the problem converges. The Benders formulation of RMCDP was presented in this paper and tested by a real instance. Moreover, the trends of objective function, best solution as well as elapsed time over iterations were illustrated. This paper aimed to show how Benders decomposition can be implemented in RMCDP to obtain an optimum solution or near optimum in a practical time. The future research could involve improving the convergence rate for larger problems by devising hybrid methods to minimize the effect of combinatorial aspect of the RMCDP.

\section{Acknowledgement}

NICTA is funded by the Australian Department of Communications and the Australian Research Council through the ICT Centre of Excellence program. 
The 31 st International Symposium on Automation and Robotics in Construction and Mining (ISARC 2014)

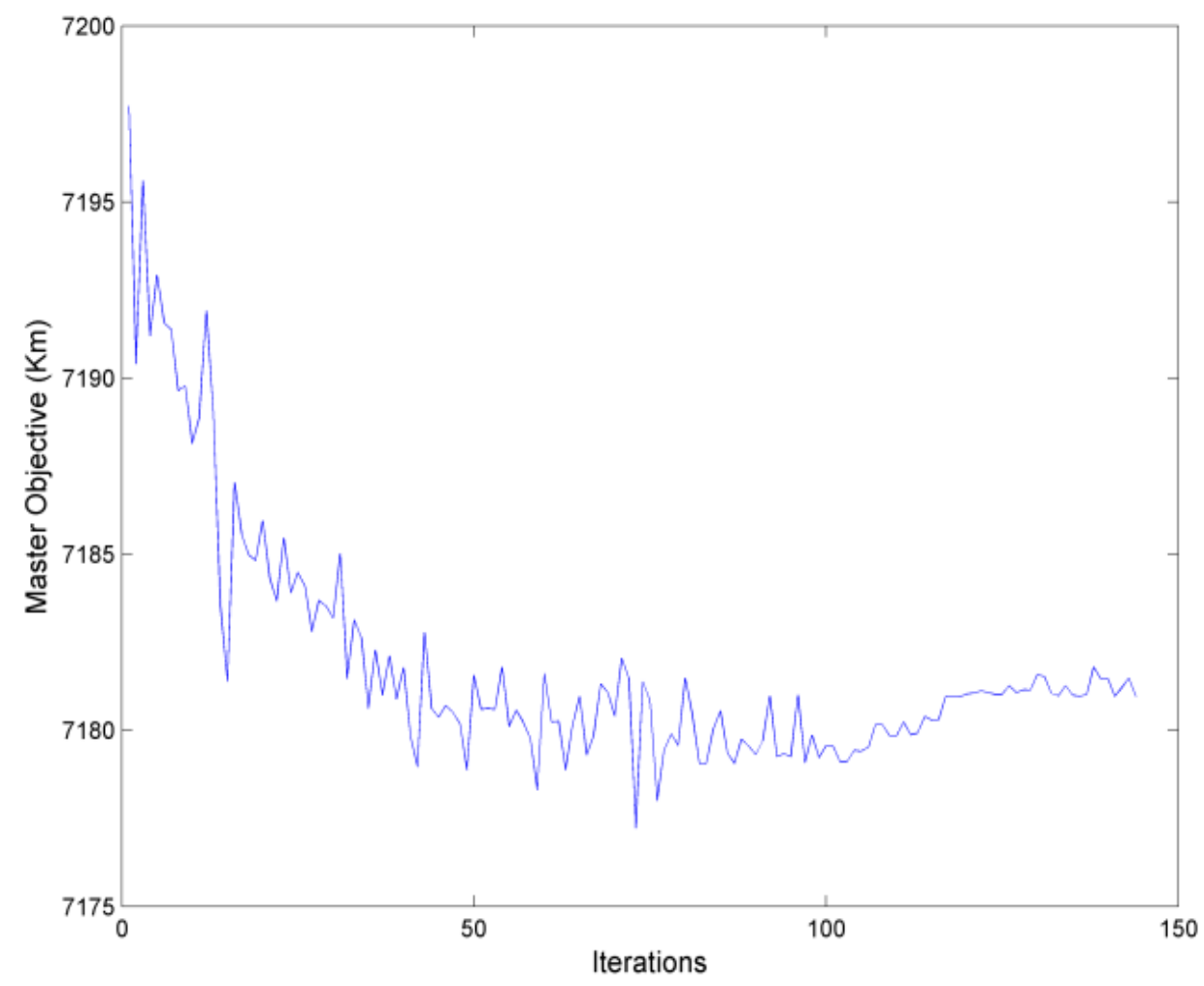

Figure 1. Objective of master problem

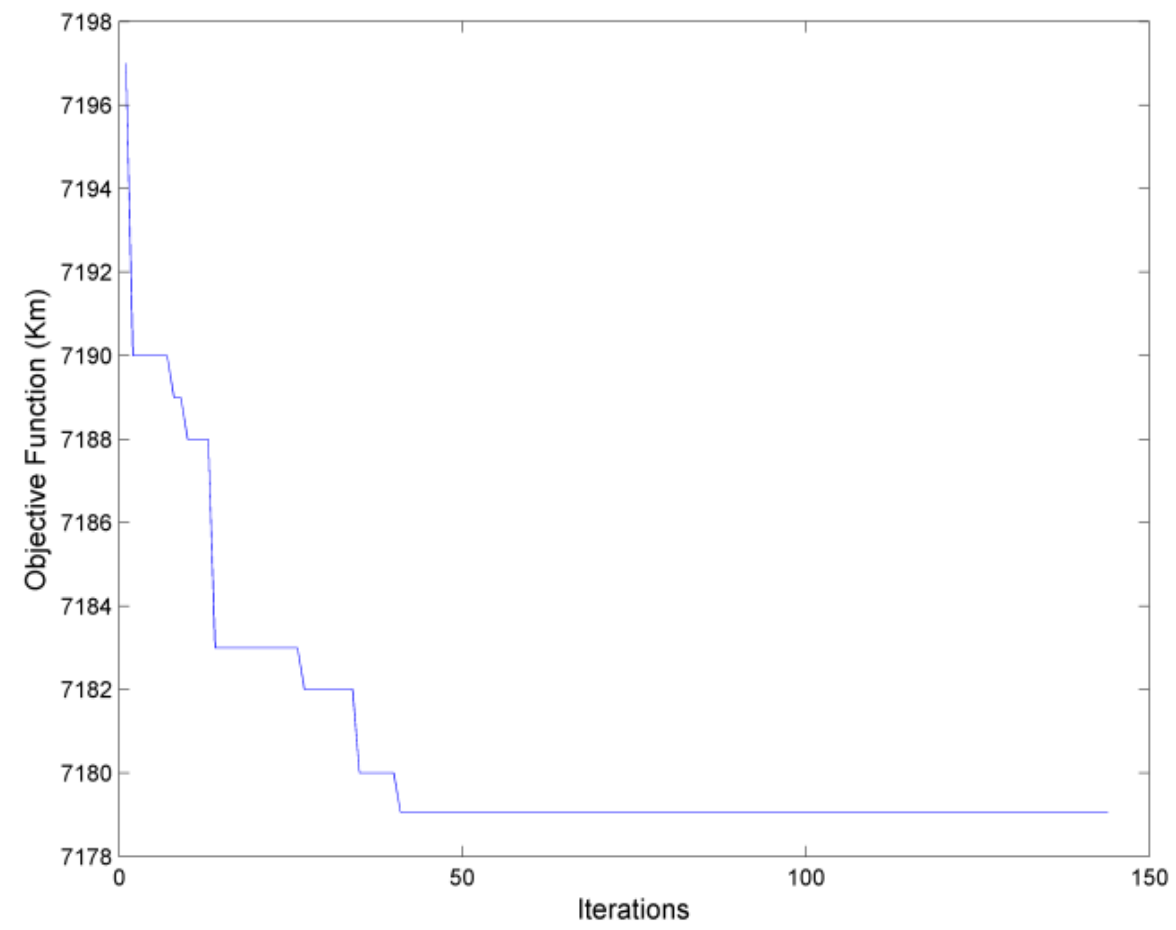

Figure 2. Best solution obtained over iterations 


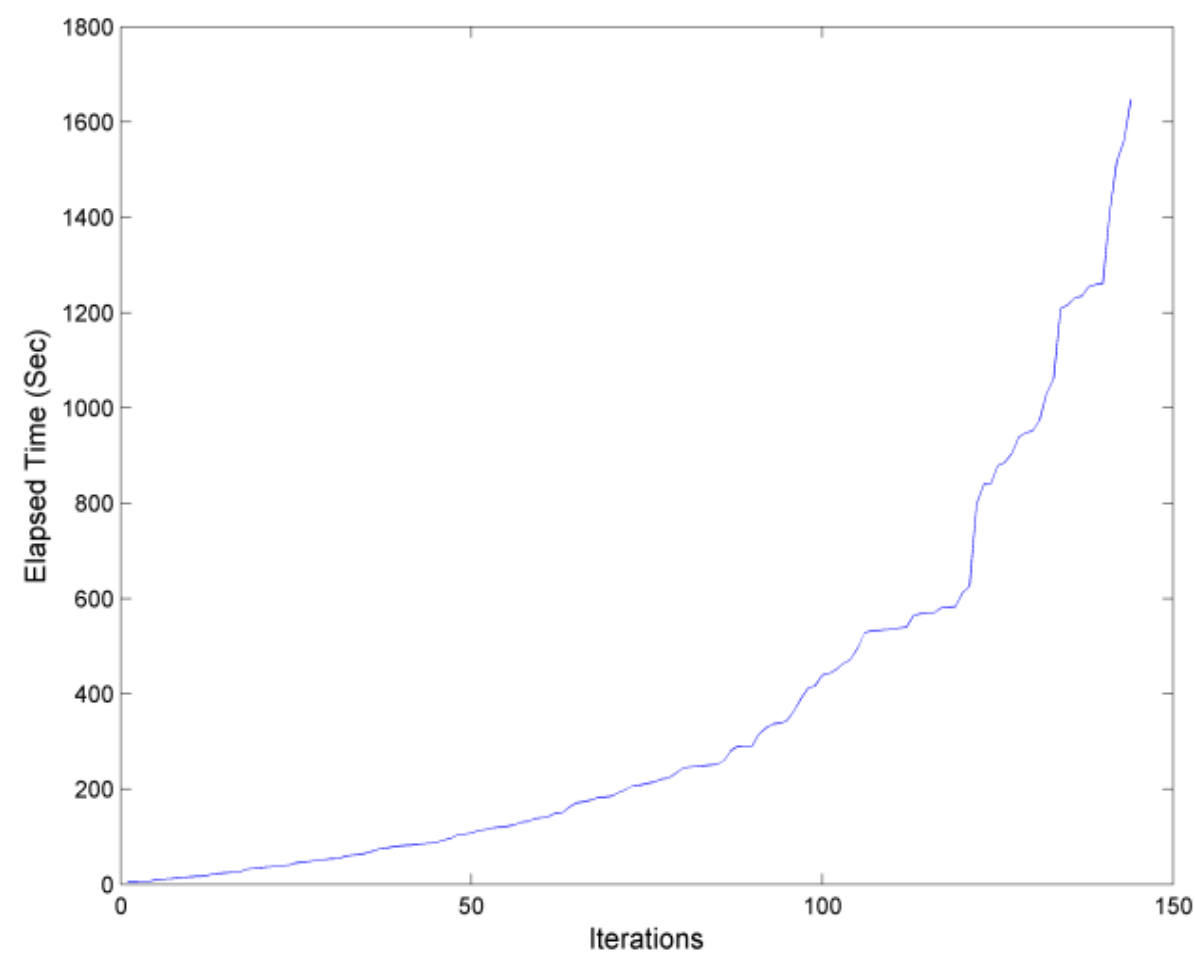

Figure 3. Elapsed time (cumulative) over iterations

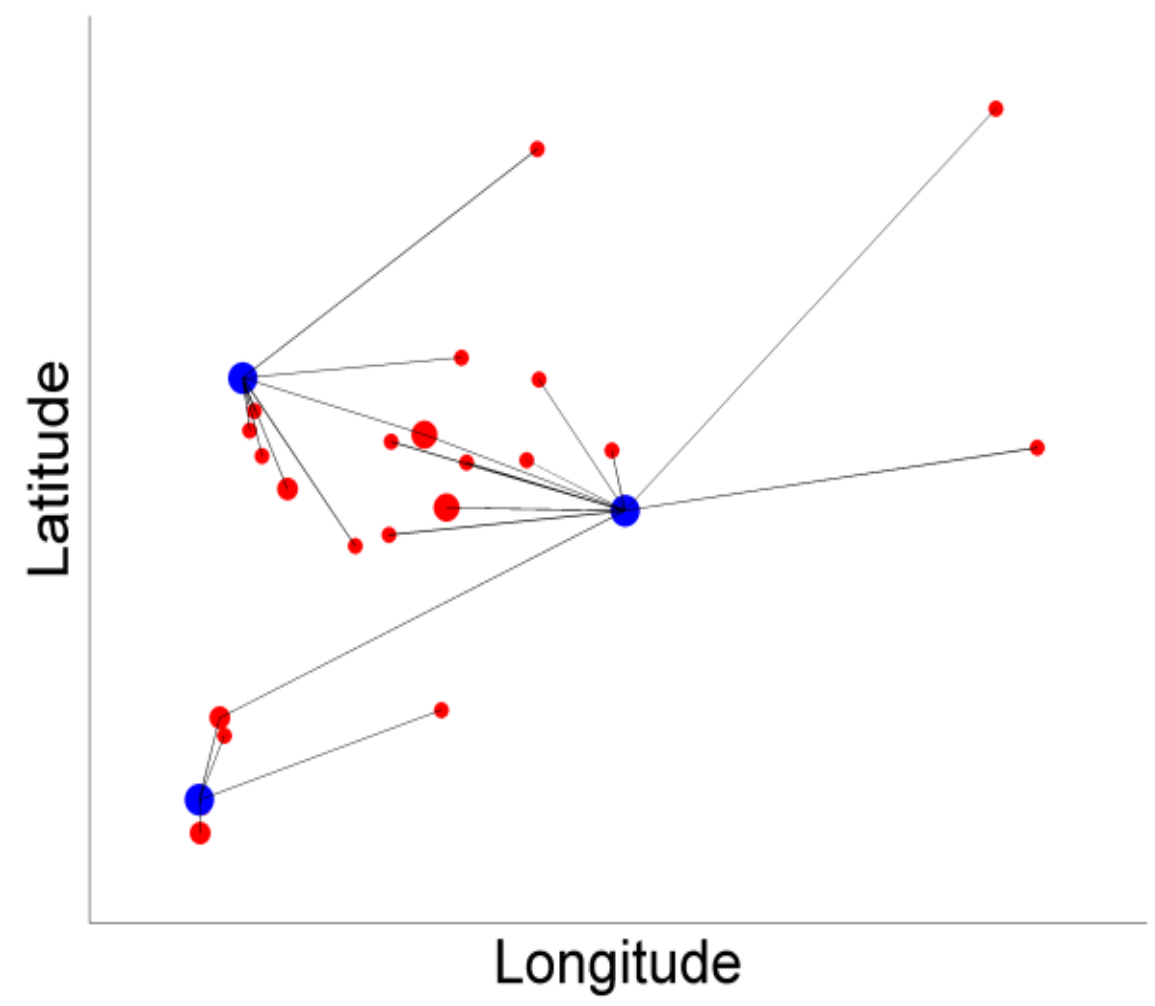

Figure 4. Travel between depots (blue dots) and customers (red dots) 


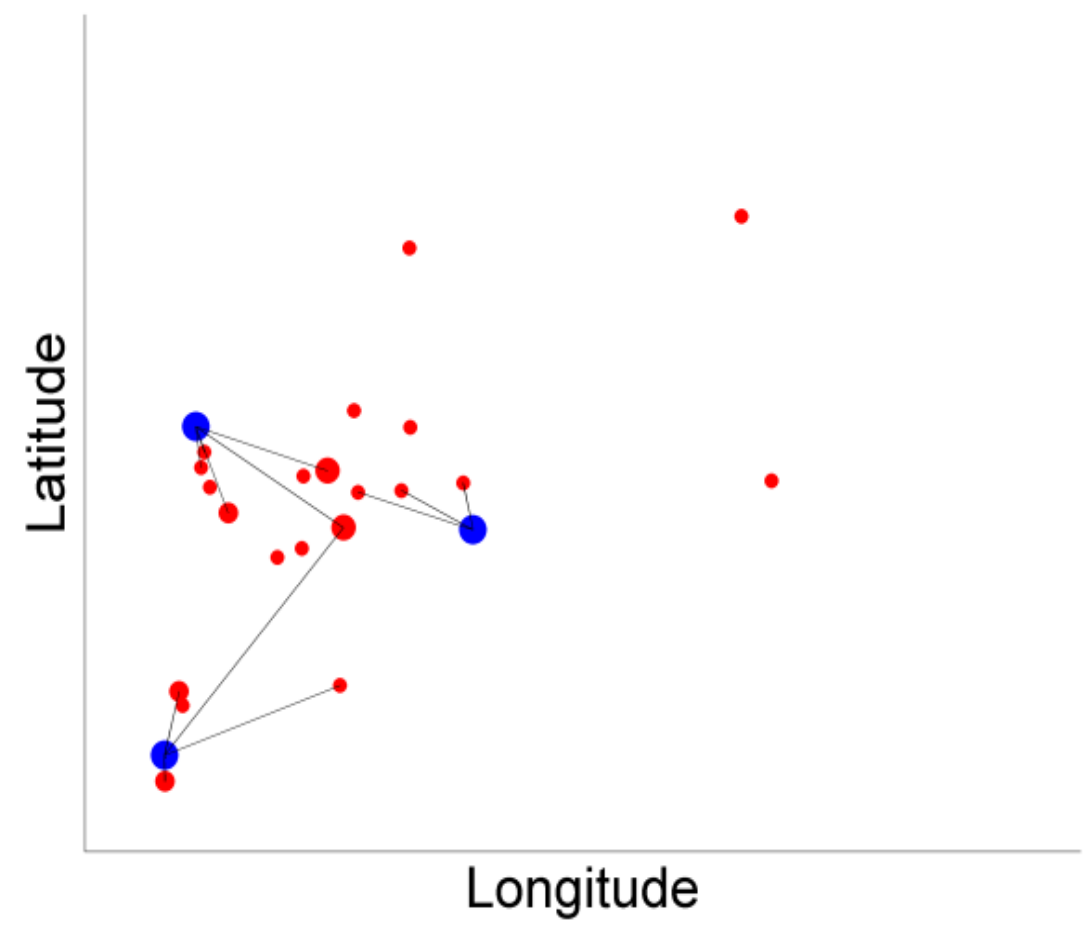

Figure 5. Travel between customers (re d dots) and depots (blue dots)

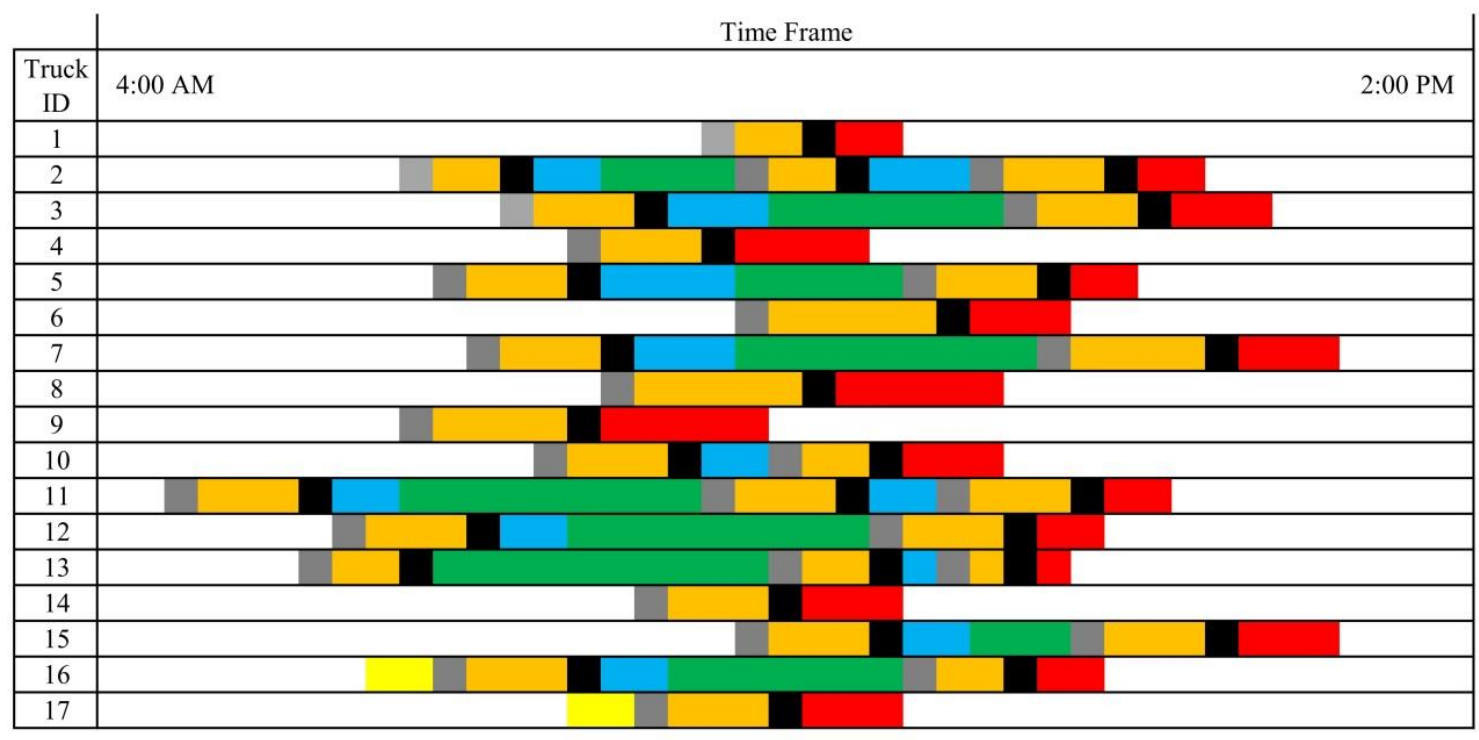

Start-> Depot

Depot- $>$ Customer

Customer $->$ Depot

Customer $->$ End

Idle Time

Unloading

Figure 6. Schedule of Trucks 


\section{Notations}

$\begin{array}{ll}\mathbf{C} & \text { Set of customers } \\ \mathbf{C}_{\mathbf{k}} & \text { Set of customers visited by a truck } \mathrm{k} \\ \mathbf{D} & \text { Set of depots } \\ \mathbf{D}_{\mathbf{k}} & \text { Set of depots visited by truck } \mathrm{k} \\ \mathbf{K} & \text { Set of vehicles } \\ \mathbf{U}_{\mathbf{s}} & \text { Set of starting points } \\ \mathbf{V}_{\mathbf{f}} & \text { Set of ending points } \\ \mathbf{S}_{\mathbf{u}} & \text { Service time at the depot u } \\ \mathbf{t}_{\mathbf{u v k}} & \text { Travel time between u and v with vehicle } \mathrm{k} \\ \mathbf{q}_{\mathbf{k}} & \text { Maximum capacity of vehicle } \mathrm{k} \\ \mathbf{q}_{\mathbf{c}} & \text { Demand of customer c } \\ \mathbf{W}_{\mathbf{u}} & \text { Time at node u } \\ \boldsymbol{\beta}_{\mathbf{c}} & \text { Penalty for not satisfying the customer c } \\ \mathbf{M} & \text { A large constant } \\ \mathbf{Y} & \text { Maximum time to haul the concrete } \\ \mathbf{X}_{\mathbf{u v k}} & \text { 1 if route between } \mathrm{u} \text { and v with vehicle } \mathrm{k} \text { is selected, 0 } \\ & \text { otherwise } \\ \mathbf{y}_{\mathbf{c}} & \text { 1 if total demand of customer c is supplied, 0 otherwise } \\ \mathbf{Z}_{\mathbf{u v k}} & \text { Cost of travel between u and v with vehicle } \mathrm{k}\end{array}$

\section{References}

[1] Feng, C.-W., T.-M. Cheng, and H.-T. Wu, Optimizing the schedule of dispatching RMC trucks through genetic algorithms. Automation in Construction, 2004. 13(3): p. 327-340.

[2] Maghrebi, M., C. Sammut, and T. Waller, Reconstruction of an Expert's Decision Making Expertise in Concrete Dispatching by Machine Learning. Journal of Civil Engineering and Architecture, 2013. 7(12): p. 1540-1547.

[3] Maghrebi, M., T. Waller, and C. Sammut. Scheduling Concrete Delivery Problems by a Robust Meta Heuristic Method. in Computer Modeling and Simulation (EMS), 2013 Seventh UKSim/AMSS European Symposium on. 2013. Manchester, UK: IEEE UK \& RI Computer Chapter.

[4] Maghrebi, M., T. Waller, and C. Sammut, Assessing the Accuracy of Expert-based Decisions in the Dispatching of Ready Mixed Concrete. ASCE Journal of construction engineering and management, 2014, 140 (6), p.1-7

[5] Naso, D., et al., Genetic algorithms for supply-chain scheduling: A case study in the distribution of ready-mixed concrete. European Journal of
Operational Research, 2007. 177(3): p. 2069-2099.

[6] Tatum, C., et al., Systems analysis of technical advancement in earthmoving equipment. Journal of construction engineering and management, 2006. 132(9): p. 976-986.

[7] Garcia, J., et al. Coordinated scheduling of production and delivery from multiple plants and with time windows using genetic algorithms. in Neural Information Processing, 2002. ICONIP'02. Proceedings of the 9th International Conference on. 2002. IEEE.

[8] Cao, M., M. Lu, and J.-p. Zhang. Concrete plant operations optimization using combined simulation and genetic algorithms. in Machine Learning and Cybernetics, 2004. Proceedings of 2004 International Conference on. 2004. IEEE.

[9] Chen, H.-K., C.-F. Hsueh, and M.-S. Chang, Production scheduling and vehicle routing with time windows for perishable food products. Computers \& operations research, 2009. 36(7): p. 2311-2319.

[10] Lu, M. and H.-C. Lam. Optimized concrete delivery scheduling using combined simulation and genetic algorithms. in Proceedings of the 37th conference on Winter simulation. 2005. Winter Simulation Conference.

[11] Silva, C.A., et al. Concrete Delivery using a combination of GA and ACO. in Decision and Control, 2005 and 2005 European Control Conference. CDC-ECC '05. 44th IEEE Conference on. 2005.

[12] Pan, L., et al. Scheduling of dispatching Ready Mixed Concrete trucks trough Discrete Particle Swarm Optimization. in Systems Man and Cybernetics (SMC), 2010 IEEE International Conference on. 2010.

[13] Lu, M. HKCONSIM: a simulation platform for planning and optimizing concrete plant operations in Hong Kong. in Proceedings of International Conference on Innovation and Sustainable Development of Civil Engineering in the 21st Century. 2002.

[14] Srichandum, S. and T. Rujirayanyong, Production scheduling for dispatching ready mixed concrete trucks using bee colony optimization. Am. J. Engg. \& Applied Sci, 2010. 3(1): p. 823-830.

[15] Yan, S., W. Lai, and M. Chen, Production scheduling and truck dispatching of ready mixed concrete. Transportation Research Part E: Logistics and Transportation Review, 2008. 44(1): p. 164-179. 
The 31st International Symposium on Automation and Robotics in Construction and Mining (ISARC 2014)

[16] Lin, P.-C., et al., Dispatching ready mixed concrete trucks under demand postponement and weight limit regulation. Automation in Construction, 2010. 19(6): p. 798-807.

[17] Yan, S., H. Lin, and X. Jiang, A planning model with a solution algorithm for ready mixed concrete production and truck dispatching under stochastic travel times. Engineering Optimization, 2012. 44(4): p. 427-447.

[18] Payr, F. and V. Schmid. Optimizing Deliveries of Ready-Mixed Concrete. in Logistics and Industrial Informatics, 2009. LINDI 2009. 2nd International. 2009. IEEE.

[19] Asbach, L., U. Dorndorf, and E. Pesch, Analysis, modeling and solution of the concrete delivery problem. European journal of operational research, 2009. 193(3): p. 820-835.

[20] Maghrebi, M., et al., Using Column Generation for Solving Large Scale Concrete Dispatching Problems 2013: UNSW Computer Science and Engineering Technical Report No. UNSW-CSETR-201334, ftp://ftp.cse.unsw.edu.au/pub/doc/papers/UNSW/20 1334.pdf.

[21] Benders, J.F., Partitioning procedures for solving mixed-variables programming problems. Numerische mathematik, 1962. 4(1): p. 238-252.

[22] Geoffrion, A.M., Generalized benders decomposition. Journal of optimization theory and applications, 1972. 10(4): p. 237-260.

[23] Feng, C.-W. and H.-T. Wu. Using genetic algorithms to optimize the dispatching schedule of RMC cars. in Proceedings of the 17th International Symposium on Automation and Robotics in Construction, Taipei, Taiwan. 2000.

[24] Feng, C.-W. and H.-T. Wu, Integrating fmGA and CYCLONE to optimize the schedule of dispatching RMC trucks. Automation in Construction, 2006. 15(2): p. 186-199.

[25] Schmid, V., et al., Hybridization of very large neighborhood search for ready-mixed concrete delivery problems. Computers \& Operations Research, 2010. 37(3): p. 559-574.

[26] Yan, S. and W. Lai, An optimal scheduling model for ready mixed concrete supply with overtime considerations. Automation in Construction, 2007. 16(6): p. 734-744.

[27] Padberg, M. and G. Rinaldi, A branch-and-cut algorithm for the resolution of large-scale symmetric traveling salesman problems. SIAM review, 1991. 33(1): p. 60-100.

[28] Dantzig, G.B., Linear programming and extensions. 1965: Princeton university press. 